\title{
Dependency of the spectral reflectance curves of the Munsell color chips
}

Jozef Cohen, UNIVERSITY OF ILLINOIS

\begin{abstract}
Abstraet
The spectral reflectance curves of 433 chips in the Munsell Book of Color have been found to depend on only three components which account for $99.18 \%$ of the variance. It is suggested that this three-component dependency may be a characteristic of all organic pigments, including those in the retina, and thus explain the trichromatic nature of color vision.
\end{abstract}

\section{Problem}

The spectral reflectance curves for 433 color chips in the Munsell Book of Color were measured with a GE recording spectrophotometer by the National Bureau of Standards; these data, in matrix form $(40 \times 417$ - the rows giving the reflectance at 40 wavelengths at $10 \mathrm{mu}$ intervals from $380 \mathrm{mu}$ to $770 \mathrm{mu}$ ), have been made available by Nickerson (1957). The columns and rows of this matrix were thought to be independent, but the computer analysis, in connection with another problem, indicated that the matrix determinant was equal to zero; the nature of this reduction in rank was investigated.

\section{Method and Results}

A $40 \times 150$ matrix of reflectance data of 150 randomly selected Munsell chips (the capacity of the computer) was subjected to a linear component analysis by the centroid method; the first four components are shown in Table 1. This application to continuous curves is almost identical to that described by Simonds (1963). Component I extracted $92.72 \%$ of the cumulative variance, component II $97.25 \%$, component III $99.18 \%$, and component IV $99.68 \%$ : considering the reduction in rank, this is one of the highest extractions of variance on record. It follows that three scalar multipliers, $M_{1}, M_{2}$, $\mathrm{M}_{3}$, may be assigned to each chip. When the first three components of Table 1 are weighted by their respective multipliers, for any chip, and each row summed, a reconstructed reflectance curve of high accuracy may be obtained for that chip. Examples of reconstructed curves with three components, and with four components, for two chips are given in Tables 2 and 3. Equally accurate reconstructions are obtained with other Munsell chips not included in this sample.

It is to be emphasized that these data are entirely physical; the three scalar multipliers do not correspond to the hue, value, and chroma of psychological specification. Given the psychological specification of a Munsell chip, the chip's reflectance curve cannot be derived; however, given the three scalar multipliers, the entire reflectance curve can be predicted with high accuracy.

\section{Diseussion}

The dependence of the Munsell reflectance curves on just three components suggests that the Munsell chips
Table 1. Four Centroid Components of Munsell Reflectance Curves

\begin{tabular}{|c|c|c|c|c|}
\hline Wavelength & $\underline{I}$ & II & III & IV \\
\hline 380 & 3.4425 & -.8360 & -.7880 & -.1795 \\
\hline 390 & 3.4776 & -.8778 & -.7860 & -.2022 \\
\hline 400 & 3.5005 & -.9140 & -.7828 & -.2284 \\
\hline 410 & 3.5145 & -.9539 & -.7776 & -.2504 \\
\hline 420 & 3.5241 & -.9937 & -.7645 & -.2659 \\
\hline 430 & 3.5366 & -1.0525 & -.7392 & -.2642 \\
\hline 440 & 3.5547 & -1.1236 & -.7088 & -.2442 \\
\hline 450 & 3.5662 & -1.2012 & -.6627 & -.2071 \\
\hline 460 & 3.5689 & -1.2788 & -.5812 & -.1628 \\
\hline 470 & 3.5561 & -1.3469 & -.4622 & -.1126 \\
\hline 480 & 3.5833 & -1.3831 & -.2469 & -.0424 \\
\hline 490 & 3.6180 & -1.3811 & .0084 & .0418 \\
\hline 500 & 3.6686 & -1.3123 & .2793 & .1201 \\
\hline 510 & 3.8025 & -1.1329 & .6102 & .2621 \\
\hline 520 & 3.9135 & -.9279 & .8886 & .4000 \\
\hline 530 & 3.9130 & -.7835 & 1.0262 & .4067 \\
\hline 540 & 3.9128 & -.6331 & 1.0998 & .3534 \\
\hline 550 & 3.9400 & -.4659 & 1.1314 & .2797 \\
\hline 560 & 3.9357 & -.2782 & 1.1241 & .2146 \\
\hline 570 & 4.0448 & -.0745 & 1.1319 & .0813 \\
\hline 580 & 4.1749 & .1705 & 1.0669 & -.0877 \\
\hline 590 & 4.4221 & .4203 & 1.0056 & -.1940 \\
\hline 600 & 4.5508 & .6630 & .7182 & -.3445 \\
\hline 610 & 4.7270 & .8696 & .4748 & -.3744 \\
\hline 620 & 4.8407 & 1.0038 & .2761 & -.3799 \\
\hline 630 & 4.8981 & 1.0793 & .1468 & -.3869 \\
\hline 640 & 4.9321 & 1.1225 & .0561 & -.3923 \\
\hline 650 & 4.9674 & 1.1465 & -.0121 & -.3868 \\
\hline 660 & 5.0134 & 1.1584 & -.0680 & -.3667 \\
\hline 670 & 5.0792 & 1.1616 & -.1198 & -.3191 \\
\hline 680 & 5.1668 & 1.1610 & -.1614 & -.2403 \\
\hline 690 & 5.2700 & 1.1553 & -.1985 & -.1285 \\
\hline 700 & 5.3864 & 1.1413 & -.2396 & .0128 \\
\hline 710 & 5.5036 & 1.1101 & -.2863 & .1719 \\
\hline 720 & 5.6102 & 1.0559 & -.3371 & .3258 \\
\hline 730 & 5.7045 & .9929 & -.3950 & .4626 \\
\hline 740 & 5.7744 & .9323 & -.4450 & .5693 \\
\hline 750 & 5.8231 & .8912 & -.4794 & .6444 \\
\hline 760 & 5.8541 & .8672 & -.5008 & .6926 \\
\hline 770 & 5.9119 & .8482 & -.5016 & .7217 \\
\hline
\end{tabular}

were created from three pigments; the Munsell chips, in fact, were produced from a large number of pigments.

Even if the Munsell chips were mixed from three pigments, it seems impossible for a linear component analysis to recover almost completely the identity of the component pigments. The formulas which relate the reflectance curves of the pigment components to the reflectance curve of the pigment mixture are complex and involve exponents (Duncan, 1949). A linear component analysis might produce a rough first approximation, but it would seem unreasonable that three linear components would exhaust the variance almost completely.

The difficulty in explaining the dependence of the Munsell chips invites speculation that the dependency is a function of the molecular structure of organic pigments, and that all organic pigments and their mixtures may be expressed as the weighted sum of three 
Table 2. Reconstruction of Spectral Reflectance, Munsell Chip R 6/6, Three and Four Components

Wavelength Measured Reconstruction Reconstruction 4

$\begin{array}{llll}380 & 31.1 \% & 29.3 \% & 30.0 \% \\ 390 & 31.1 & 29.2 & 30.1 \\ 400 & 31.0 & 29.1 & 30.1 \\ 410 & 30.7 & 28.8 & 30.0 \\ 420 & 29.1 & 28.5 & 29.7 \\ 430 & 28.1 & 28.0 & 29.1 \\ 440 & 26.9 & 27.3 & 28.4 \\ 450 & 26.0 & 26.5 & 27.4 \\ 460 & 25.3 & 25.4 & 26.1 \\ 470 & 24.3 & 24.1 & 24.5 \\ 480 & 23.6 & 22.9 & 23.1 \\ 490 & 23.0 & 22.0 & 21.8 \\ 500 & 22.8 & 21.7 & 21.3 \\ 510 & 22.7 & 23.0 & 22.0 \\ 520 & 23.1 & 24.6 & 22.9 \\ 530 & 23.1 & 25.2 & 23.6 \\ 540 & 23.5 & 26.2 & 24.9 \\ 550 & 25.0 & 27.9 & 26.9 \\ 560 & 27.4 & 29.6 & 29.0 \\ 570 & 31.5 & 32.5 & 32.6 \\ 580 & 38.2 & 36.3 & 37.3 \\ 590 & 44.9 & 41.3 & 42.7 \\ 600 & 50.5 & 46.2 & 48.3 \\ 610 & 54.8 & 51.0 & 53.2 \\ 620 & 57.4 & 54.3 & 56.5 \\ 630 & 58.8 & 56.2 & 58.4 \\ 640 & 59.4 & 57.3 & 59.6 \\ 650 & 60.0 & 58.3 & 60.4 \\ 660 & 60.3 & 59.1 & 61.1 \\ 670 & 60.7 & 60.0 & 60.8 \\ 680 & 61.4 & 61.1 & 62.4 \\ 690 & 61.8 & 62.2 & 62.9 \\ 700 & 62.2 & 63.4 & 63.4 \\ 710 & 62.7 & 64.5 & 63.6 \\ 720 & 63.0 & 65.2 & 63.6 \\ 730 & 63.3 & 65.8 & 63.5 \\ 740 & 63.6 & 66.2 & 63.1 \\ 750 & 63.9 & 66.5 & 63.1 \\ 760 & 64.2 & 67.7 & \\ 770 & 64.4 & & \end{array}$

Three Components: $\mathrm{M}_{1}=.09597, \mathrm{M}_{2}=.09192$, $\mathrm{M}_{3}=-.04994$

Four Components: $\mathrm{M}_{1}=.09623, \mathrm{M}_{2}=.09210$, $\mathrm{M}_{3}=-.04637, \mathrm{M}_{4}=-.05169$

component curves. The reflectance curves of organic pigments seem always to have a characteristic shape and belong to a family of curves; the curves, with respect to one another, seem to be neither random nor chaotic. The curves seem to be correlated. It is also true that an organic pigment may be identified uniquely by inspecting four spectrophotometric curves of the pigment dissolved in four different media (Pratt, 1947).

The problem of the generality of the dependency of organic pigments will be tested empirically but it is perhaps interesting to consider its consequences. If the general dependency should be sustained, the pigments of the eye would also be dependent on the same set of primary components. The color response curves of the retina (uncorrected for ocular media) would then be represented by the first three componets of Table 1 , and a plot should be significant. These components plot
Table 3. Reconstruction of Spectral Reflectance, Munsell Chip N 4/, Three and Four Components

Wavelength Measured Reconstruction Reconstruction

$3 \quad 4$

\begin{tabular}{|c|c|c|c|}
\hline 380 & $13.5 \%$ & $11.2 \%$ & $11.6 \%$ \\
\hline 390 & 13.3 & 11.4 & 11.8 \\
\hline 400 & 13.0 & 11.5 & 12.0 \\
\hline 410 & 12.9 & 11.7 & 12.2 \\
\hline 420 & 12.7 & 11.8 & 12.4 \\
\hline 430 & 12.6 & 12.0 & 12.6 \\
\hline 440 & 12.5 & 12.2 & 12.8 \\
\hline 450 & 12.5 & 12.4 & 12.9 \\
\hline 460 & 12.4 & 12.6 & 13.0 \\
\hline 470 & 12.2 & 12.8 & 13.0 \\
\hline 480 & 12.0 & 13.0 & 13.0 \\
\hline 490 & 11.9 & 13.1 & 13.0 \\
\hline 500 & 11.9 & 13.1 & 12.9 \\
\hline 510 & 12.0 & 13.2 & 12.6 \\
\hline 520 & 12.0 & 13.0 & 12.1 \\
\hline 530 & 12.1 & 12.7 & 11.8 \\
\hline 540 & 12.2 & 12.4 & 11.7 \\
\hline 550 & 12.2 & 12.1 & 11.6 \\
\hline 560 & 12.2 & 11.6 & 11.3 \\
\hline 570 & 12.1 & 11.4 & 11.5 \\
\hline 580 & 12.1 & 11.2 & 11.7 \\
\hline 590 & 12.1 & 11.3 & 12.1 \\
\hline 600 & 12.0 & 11.0 & 12.2 \\
\hline 610 & 12.0 & 11.0 & 12.2 \\
\hline 620 & 11.9 & 10.9 & 12.1 \\
\hline 630 & 12.0 & 10.9 & 12.1 \\
\hline 640 & 11.9 & 10.8 & 12.0 \\
\hline 650 & 11.9 & 10.9 & 12.0 \\
\hline 660 & 11.9 & 10.9 & 12.0 \\
\hline 670 & 11.9 & 11.1 & 12.1 \\
\hline 680 & 11.9 & 11.3 & 12.1 \\
\hline 690 & 11.8 & 11.6 & 12.0 \\
\hline 700 & 11.8 & 12.0 & 12.0 \\
\hline 710 & 11.8 & 12.3 & 11.9 \\
\hline 720 & 11.9 & 12.8 & 11.9 \\
\hline 730 & 11.9 & 13.2 & 11.9 \\
\hline 740 & 11.9 & 13.5 & 11.9 \\
\hline 750 & 11.9 & 13.7 & 11.9 \\
\hline 760 & 11.9 & 13.8 & 11.9 \\
\hline 770 & 11.9 & 14.0 & 12.0 \\
\hline
\end{tabular}

Three Components: $\mathrm{M}_{1}=.02728, \mathrm{M}_{2}=-.02342$, $\mathrm{M}_{3}=.00219$

Four Components: $\mathrm{M}_{1}=.02743, \mathrm{M}_{2}=-.02332$, $\mathrm{M}_{3}=.00412, \mathrm{M}_{4}=-.02792$

as a near-perfect helix (it is best to plot axes II and III, and visualize I), where complementary wavelengths seem to be opposite each other. It is odd that the Munsell reflection data should collapse to rank three, and perhaps odder still that the vectors plot in a structure so elegant.

\section{Referenees}

DUNCAN, D. R. The colour of pigment mixtures. J. Oil Colour Chem. Ass., 1949, 32, 296-321.

NICKERSON, D. Spectrophotometric Dat a for a Collection of Munsell Samples. Washington, D. C.: U.S. Department of Agriculture, 1957.

PRATT, L. S. The Chemistry and Physics of Organic Pigments. New York: Wiley, 1947. Pp. 305.

SIMONDS, J. L. Application of characteristic vector analysis to photographic and optical response data. J. Opt. Soc. Amer., 1963, 53, 968-974. 